\title{
Investigation of Part Detachment Process from Printed Circuit Boards for Effective Recycling Using Particle-Based Simulation
}

\author{
Yuki Tsunazawa $^{1, *}$, Chiharu Tokoro ${ }^{2}$, Mitsuaki Matsuoka ${ }^{2}$, Shuji Owada $^{2}$, Hiroyuki Tokuichi $^{3}$, \\ Masamichi Oida ${ }^{4}$ and Hirobumi Ohta ${ }^{5}$ \\ ${ }^{1}$ Mineral Resources Research Group, Institute for Geo-Resources and Environment, Geological Survey of Japan, National Institute of \\ Advanced Industrial Science and Technology, Tsukuba 305-8569, Japan \\ ${ }^{2}$ Department of Resources and Environmental Engineering, Faculty of Science and Engineering, Waseda University, \\ Tokyo 169-8555, Japan \\ ${ }^{3}$ Miike Smelting Co., Ltd, Omuta 836-0017, Japan \\ ${ }^{4}$ Copper Business Strategic Division, Mitsui Mining \& Smelting Co., Ltd, Tokyo 141-8584, Japan \\ ${ }^{5}$ Metals \& Recycling Division, Metals Sector, Mitsui Mining \& Smelting Co., Ltd, Tokyo 141-8584, Japan
}

For the establishment of appropriate recycling process, the delamination of electric components from printed circuit boards (PCBs), which are major components of e-waste, is expected, because some of useful critical metals are concentrated in specific components. However, the knowledge about the mechanism of the delamination process in a drum typed agitation mill is still limited and uncertain. To better comprehend the mechanism, in this study, the particle-based simulation and comminution tests using handmade PCBs were conducted. The behavior of PCBs and the air flow in the mill having flexible chains was simulated by the discrete element method (DEM) coupled with computational fluid dynamics (CFD). To model the shape of PCBs, the particle based rigid body model was introduced into the DEM. Since this model could not directly simulate the breakage phenomena, collision energy was calculated to qualitatively evaluate the comminution performance. In addition, the simulation results were compared with the experimental comminution tests. This study demonstrated the behavior of PCBs and the air flow in the mill, and the effect of the air to comminution process in the mill. It was also shown that the collision energy related to parts collision was better correlated with the experimental results and this correlation can be assumed using first order rate equation, which suggested part detachment was mainly brought by direct collision to parts in the mill. [doi:10.2320/matertrans.M-M2016834]

(Received April 20, 2016; Accepted August 26, 2016; Published November 25, 2016)

Keywords: discrete element simulation, computational fluid dynamics, recycling, comminution, e-waste

\section{Introduction}

The printed circuit boards (PCBs), which are used in all kinds of electronic equipment and electrical appliance, have been well known as metal resources because they generally contain various types of electronic components with valuable materials. Not only base metals like copper and aluminum but also critical metals like tantalum and gallium are used in $\mathrm{PCBs}^{1,2)}$. According to the report ${ }^{3)}$, the amount of electric wastes (e-waste) including PCBs has been rapidly increasing. From the viewpoint of the economy, only precious and base metals like gold, platinum, silver, ferric, copper and aluminum, has been recycled using a smelting process. In this process, it is not economically and environmentally viable to recover critical metal such as tantalum from slag because most of critical metals are incorporated in slag. Therefore, recycle technology for critical metals has not been established well yet. For a stable supply of not only precious and base metals but also these critical metals, efficient and environmental-friendly recycling process for critical metals has been strongly desired.

For the effective recovery of critical metals from PCBs, it is necessary to establish appropriate comminution and separation technology. It is known that some of critical metals are concentrated in specific electronic components, for example, $\mathrm{Ta}, \mathrm{Mn}$ and Ag in tantalum capacitors. A high-accuracy separation method especially for tantalum capacitors has been proposed by Oki et $a l^{4}$. The optimum design for orifice and

*Corresponding author, E-mail: tsunazawa-y @ aist.go.jp device has been also investigated using numerical simulation $^{5-7)}$. For the high efficiency of separation process, the delamination of electric components from PCBs is necessary. Owada et al. ${ }^{8)}$ have reported that drum typed agitation mill is effective for the parts detachment. Despite that, the mechanism of parts detachment and the delamination of electric components in the drum typed mills are still uncertain.

Since recent remarkable development of the computer hardware has made numerical simulation a strong tool of investigating various phenomena, our previous studies employed a numerical simulation to investigate the behavior of PCBs in the mill. In this numerical study, we adopted the discrete element method (DEM) $)^{9)}$, which is one of the numerical simulations of discontinuous granular flows. Since the DEM enables us to investigate the granular flow characteristics at the particle level, the DEM has been widely applied in industrial applications ${ }^{5-7,10-12)}$. In addition, we also have introduced the particle-based rigid body model ${ }^{13)}$ to the DEM for the modeling of the shapes of PCBs ${ }^{14)}$. We have confirmed the adequacy of this numerical modeling of PCBs by comparing between the simulation and experimental results. However, our numerical investigation has not taken into account of the effect of the air in the mills.

For an appropriate and effective recycling process, the objective of this study was to investigate parts detachment process from PCBs in detail, especially considering the effect of the air in the mill. The behavior of PCBs and the air flow in the drum typed agitation mill having flexible chains were calculated using the DEM coupled with computational fluid dynamics (CFD). To evaluate the comminution performance of 
the drum typed agitation mill, collision energy was calculated because this particle-based rigid body model could not directly simulate the breakage and/or deformation of PCBs. In addition, the simulation results were compared with the experimental comminution tests using handmade PCBs where some capacitors were solder-mounted and the correlation between the simulations and the experiments was also investigated.

\section{Materials and Methods}

\subsection{Drum typed agitation mill}

In this study, the Cross flow shredder (S-1000, Sato Tekko Corporation, Japan), which is a drum typed impact agitation mill, was used. The mill was a cylinder whose diameter and height were $1.00 \mathrm{~m}$, respectively. Two iron chains which had 7 rings were installed at the bottom of the mill and worked as an agitator.

\subsection{Experimental procedure}

To compare between simulation and experimental results by uniform sample, in this study, a handmade PCB was prepared. This handmade PCB was made of a double side glass epoxy PCB (FR-4, Picotec International, Taiwan), and ceramic condensers (GP075F106Z, Taiyo, Yuden, Japan). 9 ceramic condensers were solder-mounted on each PCB. The size and weight of the PCB were $210 \times 155 \mathrm{~mm}$ and $92.8 \mathrm{~g}$, respectively.

In the comminution test, two chains were initially rotated at the fixed speed. Then, 50 PCBs were inserted to the mill, and were comminuted for 30 seconds. The rotation speed of the chains was changed from 375.0, 565.5, and $750.0 \mathrm{rpm}$. After comminution, the number of ceramic condensers remained on the boards and the number of the broken boards were counted to evaluate the parts detachment and the board breakage. In this study, the PCB less than $90.0 \mathrm{~g}$ was defined as a broken board.

\subsection{Numerical simulation}

\subsubsection{Numerical modeling}

In this study, PCBs and rings were assumed as rigid objects and were modeled by a particle-based rigid body model, which can be easily introduced to the original DEM. In this model, rigid objects were modeled by many fine particles. To calculate both rigid objects and air, CFD was coupled with the DEM with a particle-based rigid body model. In this section, the modeling of rigid objects and air was briefly described.

\section{(1) Modeling of rigid objects behavior}

The motion of rigid objects is modeled according to the Newton's second law of motion. The governing equations of the translation and rotation are expressed by,

$$
\begin{gathered}
\frac{d \boldsymbol{P}}{d t}=\boldsymbol{F}^{C}+\boldsymbol{F}^{G}+\boldsymbol{F}^{D}+\boldsymbol{F}^{P G}, \\
\frac{d \boldsymbol{L}}{d t}=\boldsymbol{T}
\end{gathered}
$$

where $\boldsymbol{P}$ is the momentum of the rigid body, $\boldsymbol{F}^{\boldsymbol{C}}, \boldsymbol{F}^{\boldsymbol{G}}, \boldsymbol{F}^{\boldsymbol{D}}$ and $F^{P G}$ are the contact force, gravitational force, fluid drag force and force due to pressure gradient, $\boldsymbol{L}$ is the angular momen- tum of the rigid body, and $\boldsymbol{T}$ is torque, respectively. In this model, the contact force acting on the rigid body is calculated by the sum of the contact force acting on its fine particles because the rigid body was modeled by many fine particles. The particle-particle and particle-wall contact forces are modeled using the Voigt model, which is composed of a linear spring, dashpot and friction slider. The fluid drag force exerted on the rigid body can be obtained by integrating the fluid drag force over the volume of the fine particle which composes the rigid body.

\section{(2) Modeling of air flow}

The governing equations of the gas phase are the fluid continuity and Navier-Stokes equations for an incompressible fluid. These are expressed as,

$$
\begin{gathered}
\nabla \cdot u=0, \\
\frac{\partial(\rho \boldsymbol{u})}{\partial t}+\nabla \cdot(\rho \boldsymbol{u} \boldsymbol{u})=-\nabla p+\mu \nabla \cdot\left[\nabla \boldsymbol{u}+(\nabla \boldsymbol{u})^{\mathrm{T}}\right] \\
+\rho \boldsymbol{g}+\boldsymbol{f}^{\boldsymbol{D}},
\end{gathered}
$$

where $\boldsymbol{u}$ is fluid velocity, $p$ is pressure, $\rho$ is fluid density, $\mu$ is fluid viscosity, $\boldsymbol{f}^{\boldsymbol{D}}$ is drag force and $\boldsymbol{g}$ is gravity acceleration respectively. The drag force is the interaction force between particles and fluid. In this study, this interaction force is modelled by the immersed boundary model ${ }^{15}$. This is expressed in the form

$$
\boldsymbol{f}^{\boldsymbol{D}}=\frac{\alpha(\boldsymbol{v}-\boldsymbol{u})}{\Delta t},
$$

where $\boldsymbol{v}$ is particle velocity and $\alpha$ is local volume fraction of particles respectively. Note that the drag force is zero in the fluid domain where $\alpha=0$.

\section{(3) Calculation algorithm}

In the rigid objects, the velocity, position and angular velocity were updated using symplectic Euler scheme. In the air, a semi-implicit finite volume method employing a staggered grid was used for the discretized incompressible Navier-Stokes equation. A combination of a first order upwind scheme and a second order central scheme, which is called a hybrid scheme, was used in the convection term. The fractional step method was adopted to solve the above equations.

\section{(4) Collision energy}

In the DEM with a particle-based rigid body model, deformation and/or breakage of PCBs cannot be directly simulated because PCBs were considered as rigid bodies and inner stress of PCBs was not calculated. To estimate parts detachment and board breakage indirectly, collision energy at each collision between a particle and another particle, or a wall was calculated. Various models are available to calculate collision energy ${ }^{14,16-18)}$. In this study, the collision energy was defined as

$$
E=\frac{1}{2} \frac{m_{i} m_{j}}{m_{i}+m_{j}} v_{r}^{2},
$$

where $E$ is the collision energy, $m$ is mass of a particle, and subscripts $i$ and $j$ indicate the two collision particles, respectively. The velocity $\boldsymbol{v}_{r}$ indicate the relative velocity of collision particles at the beginning of the collision. According to this definition, the maximum collision energy was only calculated during the collision. 


\subsubsection{Numerical conditions}

\section{(1) Calculation mode}

Figure 1 illustrates a simulation model of the cross flow shredder. As mentioned above, two chains, which had 7 rings respectively, were installed at the bottom of the mill. In this study, these chain rings were considered as rigid bodies and modeled using the DEM with the particle-based rigid body model. The diameter of each constituent particle of rings was set to $6 \mathrm{~mm}$. PCBs were also modeled by a group of fine constituent particles whose diameter were $5 \mathrm{~mm}$. These constituent particles of PCBs were aligned in a grid. The model conditions are summarized in Table 1.

\section{(2) Calculation conditions}

For the reduction of computational load, in this study, a linear spring was employed and the spring constant was set a smaller value than that obtained from Young's modulus and Poisson's ratio ${ }^{19)}$. The restitution coefficient was determined in drop tests of PCBs. The sliding-friction coefficient was measured by inclined plate tests of PCBs and the rolling friction was not taken into account in this study. The physical properties are given in Table 2. In all simulations, the same parameters were used, and the calculation time was fixed at 5 seconds.

Initially, two chains were only calculated and the rotation speed of the chains gradually increased. Then, the mill was charged with PCBs, and both PCBs and the chains were calculated. During all simulations, the air was also simulated.

\section{Results and Discussions}

\subsection{Experimental results}

\subsubsection{Effect of the input number of PCBs}

To investigate the effect of the input number of PCBs, comminution tests, where the input number of PCBs was changed from 3 to 50, were conducted. In these experiments, the rotation speed of the chains was fixed at $750.0 \mathrm{rpm}$ and the com-

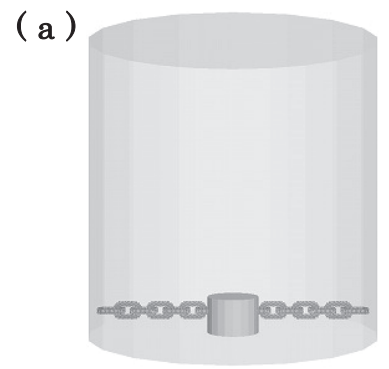

Fig. 1 Simulation model of the cross flow shredder, (a) Front view, (b) Top view.

Table 1 Model conditions in the particle based rigid body model.

\begin{tabular}{lc}
\hline PCB: & \\
Particle diameter (mm) & 5.0 \\
Number of particles for a board (-) & 1320 \\
Number of particles for a part (-) & 2 \\
Number of parts (-) & 9 \\
Mass of a PCB (g) & 92.8 \\
Ring: & 6.0 \\
Particle diameter (mm) & 342 \\
Number of particles for a ring (-) & 390.0 \\
Mass of a ring (g) & \\
\hline
\end{tabular}

minution time was set to 5 seconds. Figure 2 shows that the relationship between the input number of PCBs and parts detachment, or board breakage. As shown in this graph, both the percentage of parts detachment and the percentage of board breakage increased as the input number of PCBs. In any conditions, most PCBs were broken into fragments and most parts detached from the board. The larger the number of PCBs was, the larger the number of collision between a PCB and another PCB became. As a result, the interaction of PCBs promoted the parts detachment and the board breakage.

\subsubsection{Effect of the rotation speed of chains}

Next, to investigate the effect of the rotation speed of chains, comminution tests, where the rotation speed of the chains was changed from 375.0, 565.5, and $750.0 \mathrm{rpm}$, was conducted. In these experiments, the input number of PCBs was set at 50 and the comminution time was set to 5 seconds. Figure 3 shows that the relationship between the rotation speed of the chains and parts detachment, or board breakage. As shown in this graph, as the rotation speed of the chains, both the percentage of parts detachment and the percentage of board breakage increased. In addition, it was found that the percentages of board breakage rapidly increased whereas that of parts detachment gradually increased. Most PCBs were broken into fragments at $750 \mathrm{rpm}$ whereas most PCBs kept much of their original shapes at $375.0 \mathrm{rpm}$. This result indicated that the rotation speed of the chains affected more the board breakage than the parts detachment.

\subsection{Simulation results}

\subsubsection{Visualization of PCBs and air in the mill}

To visualize the behavior of PCBs, the chains and air in the mill, DEM-CFD including the particle-based rigid body model simulation was performed. In these simulations, the rota-

Table 2 Physical properties of solid particles and gas phase.

\begin{tabular}{lc}
\hline Solid particles: & \\
Spring constant $(\mathrm{N} / \mathrm{m})$ & $5.0 \times 10^{6}$ \\
Coefficient of restitution (-) & 0.36 \\
Coefficient of friction (-) & 0.27 \\
\hline Gas phase: & 1.0 \\
Gas density $\left(\mathrm{kg} / \mathrm{m}^{3}\right)$ & $1.8 \times 10^{-5}$ \\
Viscosity (Pa s) & \\
\hline
\end{tabular}

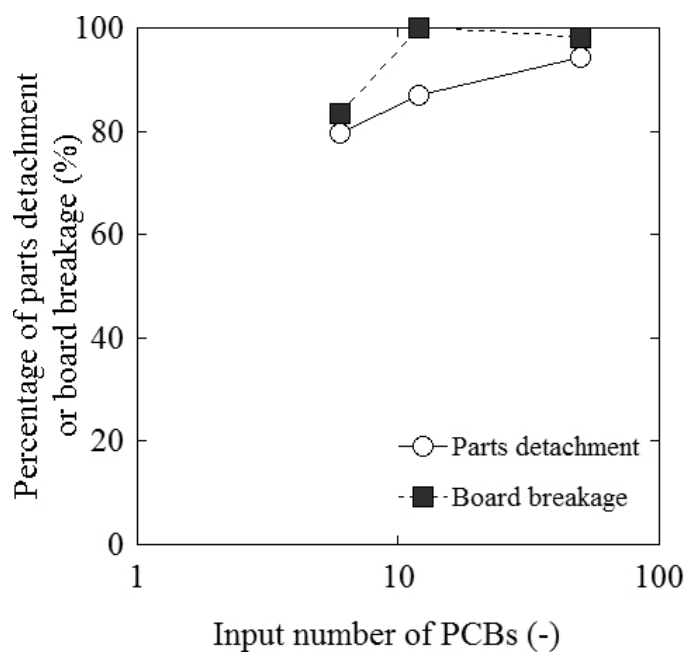

Fig. 2 Effect of the input number of PCBs in experiments. 
tion speed of the chains was fixed at the constant set up condition. First, to investigate the air flow in the mill, the chains and air were only simulated at each rotation speed of the chains. Simulation results showed that the air flow arose from the rotation of the chains in the horizontal direction. It was observed that the air flow near the chains had higher velocity and the velocity was especially high along the inner wall.

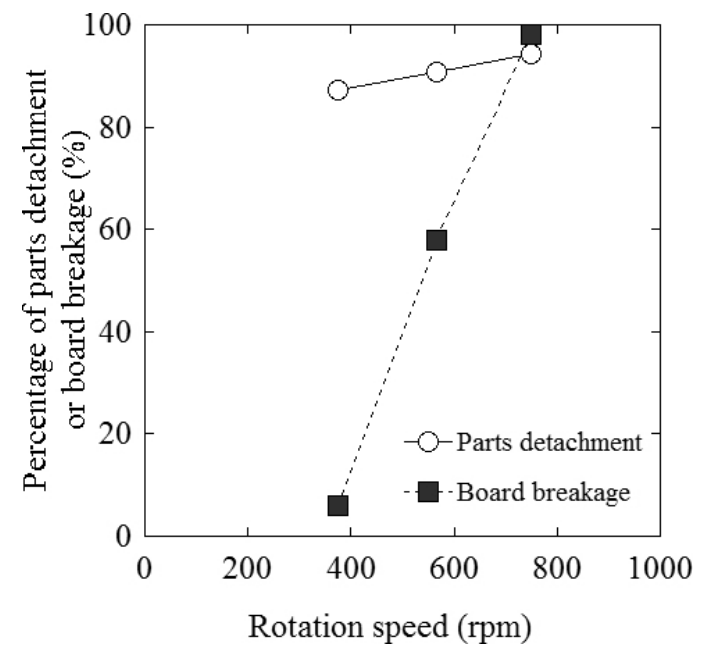

Fig. 3 Effect of the rotation speed of the chains in experiments.
This result indicated that the rotation of the chains caused the air flow at the bottom of the mill. It was also observed that the upward flow occurred near the inner wall whereas the downward flow occurred at the center of the mill in the vertical direction. The velocity of the flow in the horizontal direction was higher than that in the vertical direction.

Next, the simulations of PCBs, the chains, and the air were conducted. In these simulations, the rotation speed was fixed at the constant setup condition. Figure 4 shows typical snapshots of the behavior of PCBs and the air. It was shown that PCBs were agitated by the rotation of the chains. PCBs near the bottom of the mill had relatively high velocity because they collided with the chains and got high velocity. It was also observed that the flow pattern in the vertical direction was a little more turbulent than that with no PCBs simulation. This result indicated that the existence of PCBs affected the flow especially in the vertical direction. On the other hand, the flow pattern in the horizontal direction was almost the same as that with no PCBs simulation. This was because the flow along with the chain rotation was so strong that the existence of PCBs had little effect on it. It was also observed that the flow surrounding PCBs occurred in the vertical direction. It was suggested that the interaction between PCBs and the air flow was promoted in the mill.

\subsubsection{Evaluation of the effect of air flow}

To quantitatively evaluate the effect of air in the mill, aver-
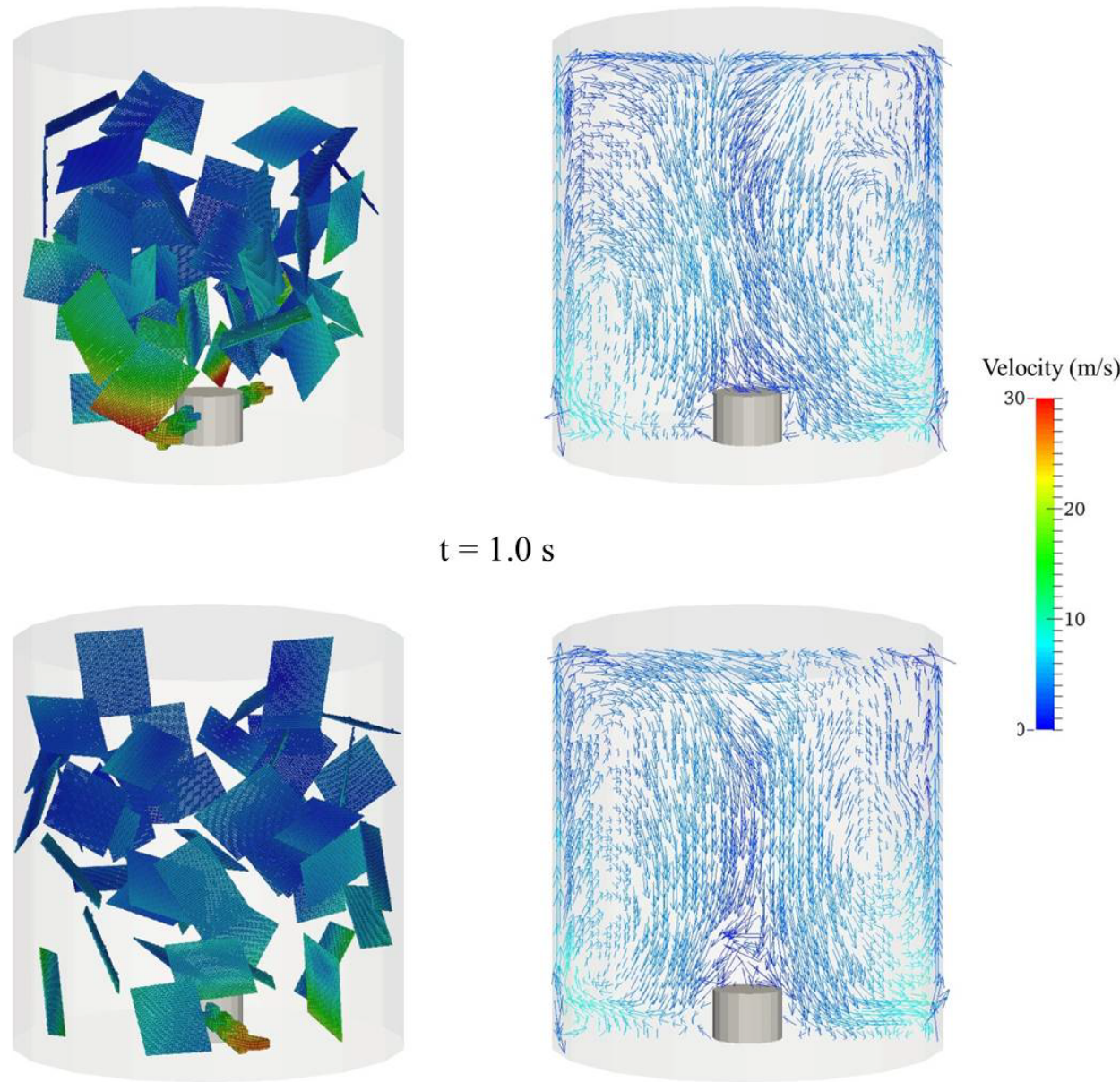

$\mathrm{t}=2.0 \mathrm{~s}$

Fig. 4 Typical snapshots of PCBs and fluid flow in Cross Flow Shredder. 
age velocity of PCBs was compared between DEM-CFD simulations (with air) and DEM simulations (without air). Figure 5 shows the average velocity distribution of PCBs at each height of the mill. As shown this graph, in any rotation speed, average velocity of PCBs obtained from DEM-CFD simulations was larger than that obtained from DEM simulations. In the bottom side, the differences of the average velocity were remarkable and resulted from the rotation of the chains. On the other hand, in the upper side, the average velocity of PCBs obtained from DEM-CFD simulations was almost the same as that obtained from DEM simulations. This was because the flow velocity in the upper side was smaller than that in the bottom side and the air flow in the upper side had little effect on PCBs.

In addition, to quantitatively evaluate the interaction between PCBs and the air flow, contact time of PCBs was compared between DEM-CFD simulations and DEM simulations. All collisions were divided into three; a collision between a PCB and another PCB, a collision between a PCB and a chain, and a collision between a PCB and a drum wall. Figure 6 shows the distribution of contact time between a PCB and another PCB in the condition where the rotation speed of the chains was $565.5 \mathrm{rpm}$. It was found that most collisions between a PCB and another PCB lasted for less than $3.0 \mathrm{~ms}$, regardless of whether air was considered or not. It was also observed that some collisions lasted for more than $4.0 \mathrm{~ms}$ in the DEM-CFD simulation. The distribution of contact time between a PCB and a chain in the condition where the rotation speed of the chains was $565.5 \mathrm{rpm}$ is shown in Fig. 7. It was shown that most collisions between a PCB and a chain lasted for less than $4.0 \mathrm{~ms}$ in both DEM-CFD and DEM simulations. It was also found that some collisions lasted for over $4.0 \mathrm{~ms}$ and the number of these collisions in DEM-CFD simulations was larger than that in DEM simulations. This result indicated that PCBs collided with the chains for a longer time in the presence of the air flow and the interaction between PCBs and the chains increased. This was because the flow surrounding PCBs increased the contact time between PCBs and the chains. Figure 8 shows the distribution of contact time between a PCB and a drum wall in the condition where the rotation speed of the chains was $565.5 \mathrm{rpm}$. It was shown

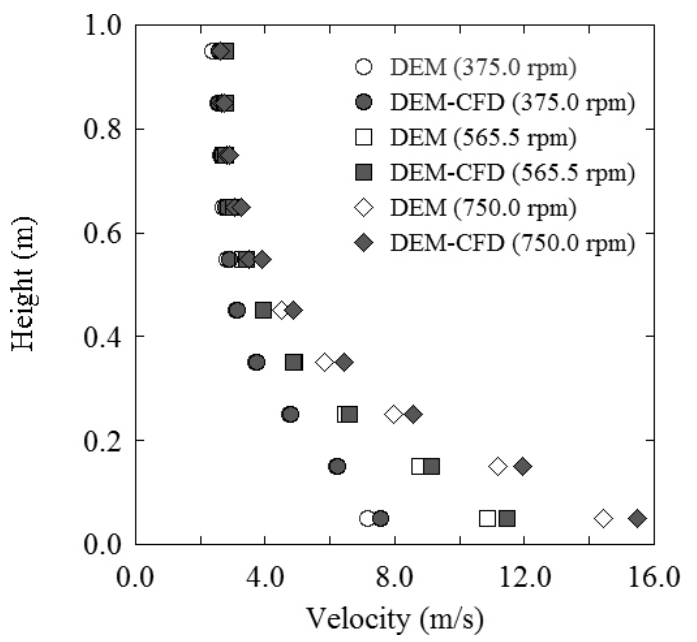

Fig. 5 Distribution of PCB's velocity at each height in Cross Flow Shredder. that most collisions between a PCB and a drum wall lasted for less than $5.0 \mathrm{~ms}$ in both DEM-CFD and DEM simulations and some other collisions lasted for over $5.0 \mathrm{~ms}$. It was also found that the number of collisions for over $5.0 \mathrm{~ms}$ in DEMCFD simulations was much larger than that in DEM simulations whereas the number of collisions for less than $5.0 \mathrm{~ms}$ in DEM-CFD simulations was smaller than that in DEM simulations. This result indicated that PCBs collided with the drum wall for a longer time in the presence of the air flow and the interaction between PCBs and the drum wall increased as well as in the case of collisions between PCBs and the chains. This was because the upward flow near the inner wall increased the contact time between PCBs and the drum wall. In the other conditions where the rotation speed of the chains was 375.0 and $750.0 \mathrm{rpm}$, almost the same trends of contact time were confirmed.

\subsection{Correlation between simulation and experiments}

The correlation between the simulations and the experiments was investigated. In the experiments, the percentage of parts detachment was counted to characterize performance of comminution in the mill. On the other hand, in the simulations, collision energy obtained from DEM-CFD simulations

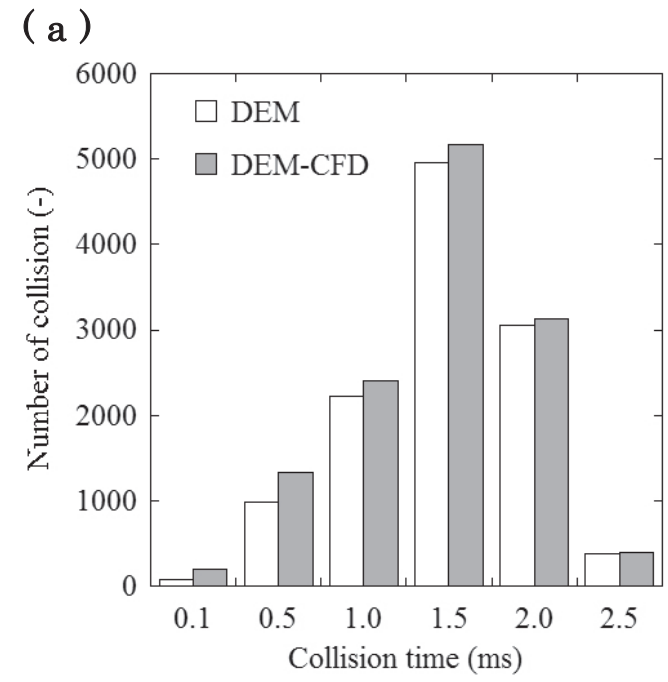

( b )

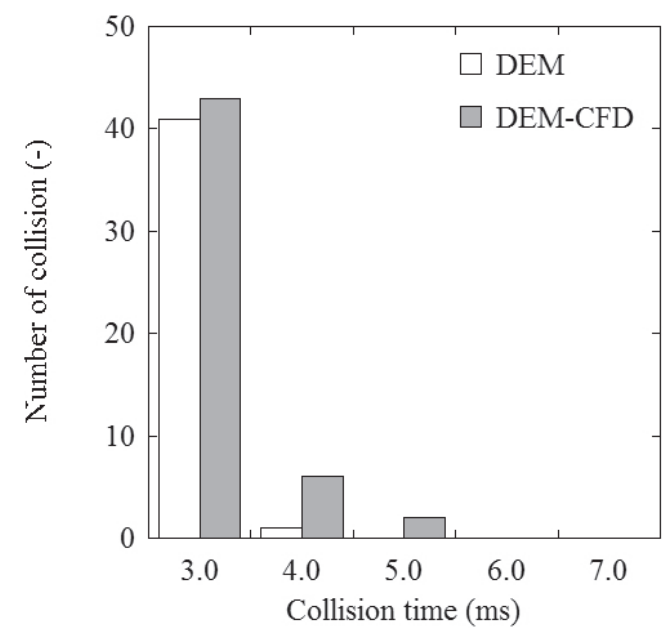

Fig. 6 Distribution of collision time between a PCB and another PCB (Chain rotation speed: $565.5 \mathrm{rpm}$ ), (a) 0.1-2.5 ms, (b) 3.0-7.0 ms over. 
( a )

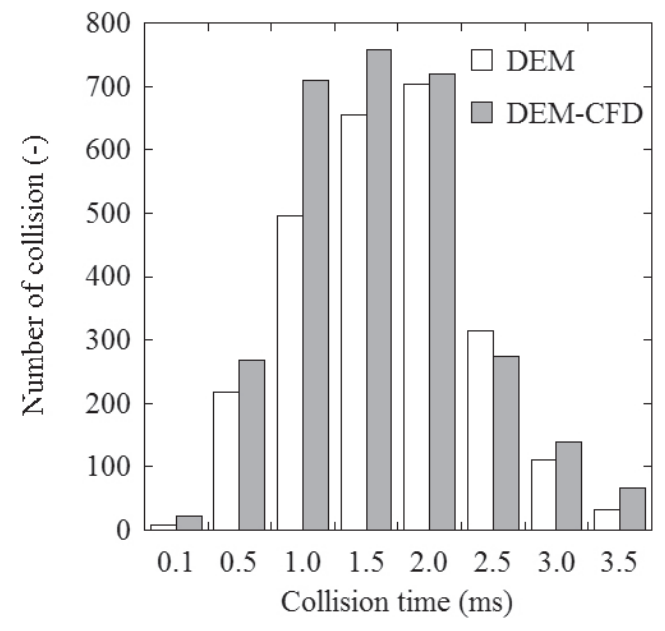

( b )

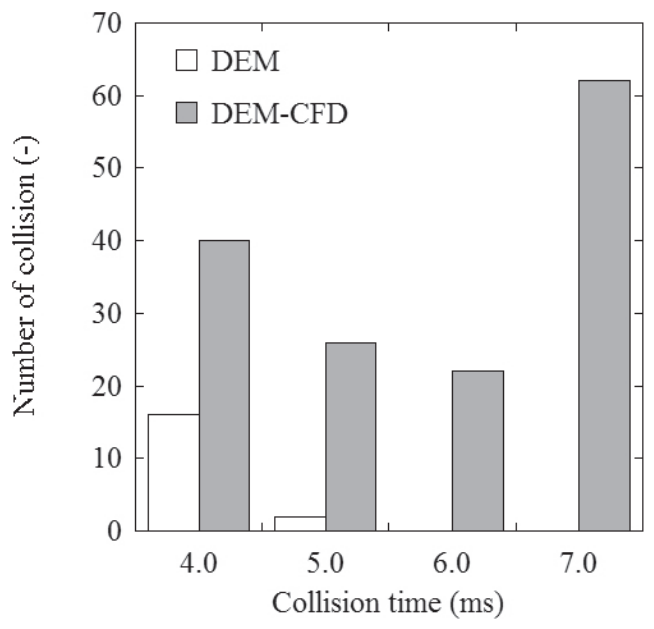

Fig. 7 Distribution of collision time between a PCB and a chain (Chain rotation speed: $565.5 \mathrm{rpm}$ ), (a) 0.1-3.5 ms, (b) 4.0-7.0 $\mathrm{ms}$ over.

was used since it cannot be negligible that the air flow in the mill had an effect on PCB's behavior. This study considered that all collisions can be divided into board collisions and parts collisions. In all collisions, collision energy was calculated based on eq. (6), and then divided into board collisions and parts collisions. After the simulations, both collisions related to boards and collisions related to parts were integrated respectively. Both total energies related to board collisions and parts collisions were respectively normalized by the input number of PCBs. These normalized collision energies were considered as an index characterizing the collisions in the simulations.

This study considered that the ratio of parts detachment rapidly increased along with the increase of the energy that PCBs are given, and the ratio of parts detachment will become saturate at certain energy. Thus, the correlation between the simulations and the experiments was assumed using first order rate equation. This correlation can be expressed in the following form

$$
-\frac{d P}{d E}=k P
$$

where $P$ is percentage of parts detachment, $k$ is kinetic con-

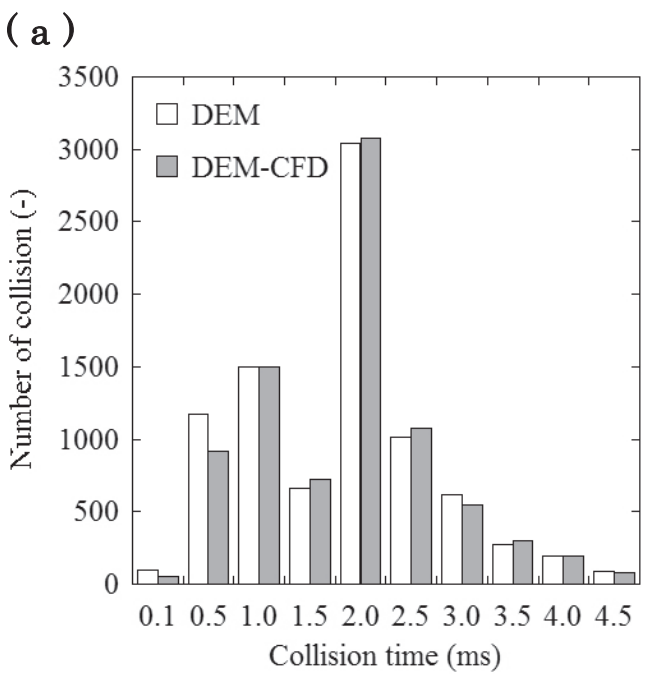

( b )

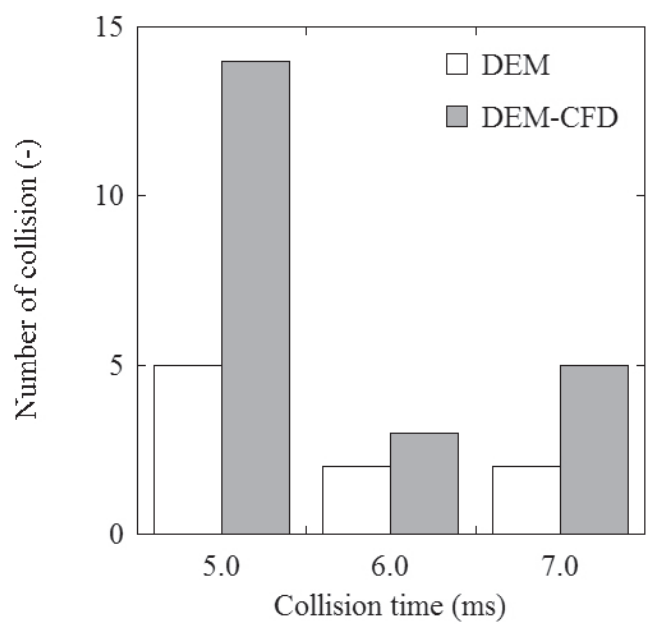

Fig. 8 Distribution of collision time between a PCB and a drum (Chain rotation speed: $565.5 \mathrm{rpm}$ ), (a) $0.1-4.5 \mathrm{~ms}$, (b) 5.0-7.0 ms over.

stant of parts detachment obtained from the experiments, and $E$ is the normalized total collision energy obtained from the simulations. The kinetic constant was a fitting parameter, which was determined from the plots obtained from the simulations and the experiments.

Figure 9 shows the correlation between the normalized collision energy and the percentage of parts detachment. In these graphs, the line shows a fitting curve based on eq (7). As shown in these graphs, it was found that the collision energy related to parts collisions was better correlated with the experimental results than that related to board collisions. These results suggested that parts detachment was obtained from direct collisions to parts in the mill, although indirect collision of boards with deformation would slightly affect it.

\section{Conclusion}

In this study, comminution process of PCBs in the drum typed agitation mill was investigated. To model the shape of PCBs and the flexible chains, the particle-based rigid body model was introduced to the DEM. In addition, to consider the air flow in the mill, the DEM with the particle-based rigid body model was coupled with the CFD. To investigate com- 
minution process, the simulation results were compared with the comminution experiments using handmade PCBs. The results obtained from this study are summarized as follows:

(1) To investigate the effect of the input number of PCBs and the rotation speed of the chains, comminution tests were conducted. As the input number of PCBs increased, the percentage of parts detachment increased. As the rotation speed of the chains increased, the percentage of parts detachment increased. These results indicated that both the input number and the rotation speed were important factors which determined the parts detachment.

(2) The behaviors of PCBs and the air flow were visualized. The air flow arose from the rotation of the chains in the horizontal direction, and in the vertical direction, the upward flow near the inner wall and the downward flow at the center of the mill occurred. On the other hand, PCBs were agitated by the rotation of the chains. It was also found that the flow surrounding PCBs occurred in the vertical direction and promoted the interaction between PCBs and the air flow.

(3) To quantitatively evaluate the interaction between PCBs and the air flow, average velocity and contact time of PCBs were compared between DEM-CFD simulations

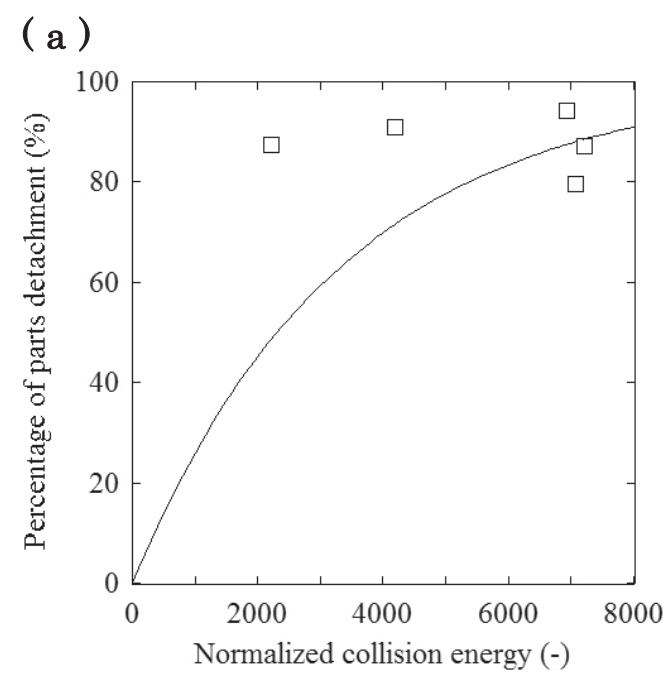

( b )

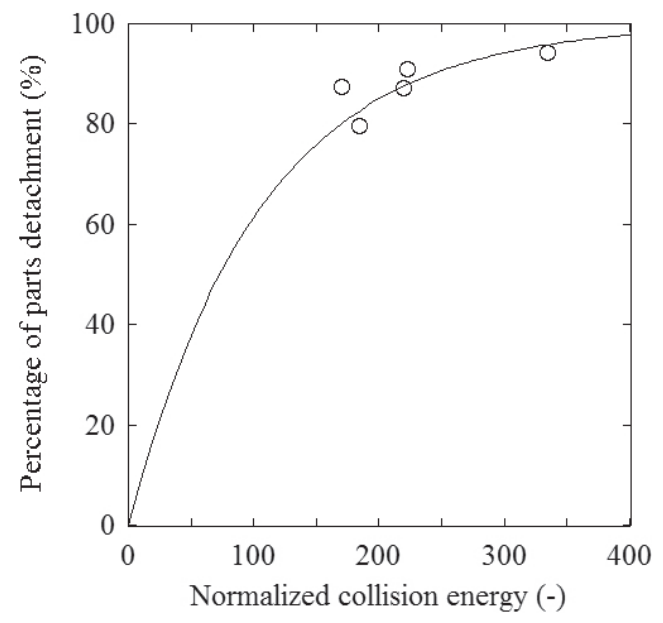

Fig. 9 Correlation between the simulations and the experiments, (a) boards, (b) parts. and DEM simulations. Average velocity obtained from DEM-CFD simulations was larger than that obtained from the DEM simulations especially at the higher rotation speed of the chains. In addition, contact time obtained from DEM-CFD simulations was longer than that obtained from DEM simulations. These results indicated that the effect of the air in the mill cannot be negligible when considering PCBs behavior by the DEM simulation.

(4) To investigate the correlation between the simulations and the experiments, the collision energy obtained from the simulations was compared with the percentage of parts detachment obtained from the experiments. It was found that the collision energy related to parts collision was better correlated with the experimental results and the correlation could be assumed using first order rate equation. These results suggested part detachment was mainly brought from direct collision to parts in the mill.

\section{Acknowledgments}

This research was partially supported by the Japan Society for the Promotion of Science (JSPS). This research was also partially supported by the Development and Demonstration of Priority Rare Metal Collection and Recycling technology of the Ministry of Economy, Trade and Industry (METI) and the Environmental Research and Development Fund (3K153009) of the Ministry of the Environment (MOE).

The authors are deeply grateful to Sato Tekko Co. for their special support for our experiments.

\section{REFERENCES}

1) T. Yumoto and T. Shiratori: J. MMIJ 125 (2009) 75-80.

2) T. Yumoto, T. Shiratori and T. Nakamura: J. MMIJ 126 (2010) 95-102.

3) S. Herat: Clean Soil Air Water 35 (2007) 305-310.

4) T. Oki, H. Yotsumoto and N. Ishida: AIST Annual Report, (AIST, Tokyo, 2005) p. 268

5) N. Hayashi and T. Oki: Mater. Trans. 55 (2014) 700-707.

6) N. Hayashi and T. Oki: Mater. Trans. 55 (2014) 952-957.

7) N. Hayashi and T. Oki: Mater. Trans. 56 (2015) 140-148.

8) S. Owada, C. Koga, S. Kageyama, C. Tokoro, T. Shiratori and T. Yumoto: J. MMIJ 128 (2012) 626-632.

9) P.A. Cundall and O.D.L. Strack: Geotechnique 29 (1979) 47-65.

10) H. Mio, J. Lee, T. Nakagawa, J. Kano and F. Saito: Mater. Trans. 42 (2001) 2460-2464

11) Y. Tsunazawa, Y. Shigeto, C. Tokoro and M. Sakai: Chem. Eng. Sci. 138 (2015) 791-809.

12) Y. Tsunazawa, D. Fujihashi, S. Fukui, M. Sakai and C. Tokoro: Adv. Powder Technol. 27 (2016) 652-660.

13) M. Tanaka, M. Sakai and S. Koshizuka: Trans. of JSCES (2007) 20070007.

14) Y. Tsunazawa, K. Tahara, K. Hosoda, C. Tokoro and S. Owada: J. Soc. Powder Technol. Japan 51 (2014) 415-423.

15) T. Kajishima, S. Takiguchi, H. Hamasaki and Y. Miyake: JSME Int. J. Ser. B. 44 (2001) 526-535.

16) J. Park and C.R. Wassgren: Particul. Sci. Technol. 21 (2003) 157-175.

17) H. Mio, J. Kano, F. Saito and M. Ito: J. MMIJ. 123 (2007) 97-102.

18) H. Nakamura, H. Fujii and S. Watano: Powder Technol. 236 (2013) 149-156.

19) C. Tokoro, K. Okaya and J. Sadaki: J. Soc. Powder Technol. Japan. 40 (2003) 236-245. 